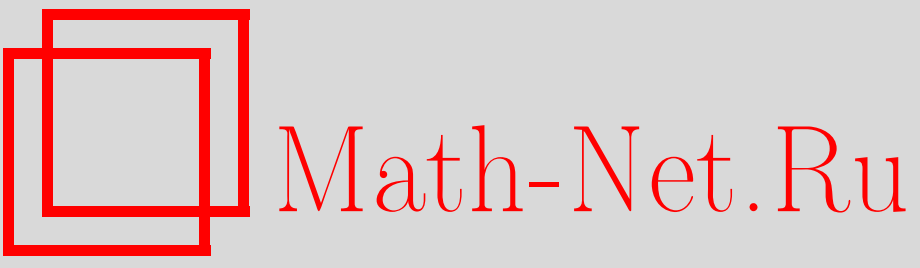

Р. В. Михайлов, О невидимых подгруппах, УМН, 2002, том 57, выпуск 6, 187-188

DOI: https://doi.org/10.4213/rm585

Использование Общероссийского математического портала Math-Net.Ru подразумевает, что вы прочитали и согласны с пользовательским соглашением

http://www.mathnet.ru/rus/agreement

Параметры загрузки:

IP : 54.172 .240 .79

26 апреля 2023 г., $17: 37: 54$ 


\title{
О НЕВИДИМЫХ ПОДГРУППАХ
}

\author{
Р. МихАЙлов
}

Нормальная подгруппа $K$ группы $G$ назьвается невидимой в $G$, если $K$ является нормальньм замьканием конечного множества элементов из $G$ и $[K, G]=K$ (см. [1]). Невидимые подгруппы возникают при изучении алгебраических замыканий групп и алгебраических вопросов, связанных с инвариантами конкордантности классических зацеплений. В частности, если $K$ - невидимая подгруппа в $G$ и $\phi: G \rightarrow A$ - некоторый гомоморфизм, где $A$ - алгебраически замкнутая группа, то $K \subset \operatorname{Ker} \phi$.

Для произвольной группы $G$ рассмотрим ее трансфинитньй нижний центральньй ряд, определяемьй индуктивно: $\gamma_{1} G=G, \ldots, \gamma_{\tau+1} G=\left[\gamma_{\tau} G, G\right]$ для любого ординала $\tau$ и $\gamma_{\tau} G=$ $\bigcap_{\tau_{i}<\tau} \gamma_{\tau_{i}} G$, если $\tau$ является предельным ординалом последовательности $\tau_{i}, i=1,2, \ldots$, т.е. $\tau$ - наименьший ординал со свойством $\tau_{i}<\tau$ для всех $i$. Длиной нижнего центрального ряда группы $G$ (или просто длиной) называется наименьшее $\tau$ такое, что $\gamma_{\tau} G=\gamma_{\tau+1} G$.

В работе [2] поставлена следующая проблема: пусть $G$ конечно-порожденная группа длины $\alpha$, верно ли, что $\gamma_{\alpha} G$ представляется в виде объединения невидимых подгрупп? Для бесконечно-порожденных групп легко строятся контрпримеры [2], а для конечно-порожденных групп, понятно, имеет смысл рассматривать лишь бесконечные ординалы, так как для конечных $n \gamma_{n} G$ является нормальньм замыканием конечного числа коммутаторов веса $n$.

Основным результатом данной работы является следующий пример, решающий проблему из [2].

Teорема. Группа $G=\left\langle a, b \mid\left[a, a^{b^{i}}\right]=\left[a, a^{a^{b^{i+1}}}\right] ; i=1,2, \ldots\right\rangle$ имеет длину строго $\omega$, но $\gamma_{\omega} G$ не представляется в виде обвединения невидимых подгрупп ${ }^{1}$.

Лемма 1. В свободной группе ранга $2 F_{2}=\langle a, b\rangle$ имеет место включение

$$
\left[a, a^{g}\right] \in\left\langle\left[a, a^{b^{i}}\right], i=1,2, \ldots\right\rangle^{F_{2}}
$$

для любого $g \in F_{2}$.

Лемму 1 можно доказать, например, с помощью выбора свободного базиса в подгруппе $\left\langle\left[a, a^{g}\right] \mid g \in F_{2}\right\rangle^{F_{2}}$ или просто рассматривая разложение элемента $g$ в базисе группы $F_{2}$ и применяя коммутаторные соотношения. Далее везде $G$ будет обозначать группу из теоремы.

Лемма 2. $\gamma_{\omega} G=\left\langle\left[a, a^{b^{i}}\right], i=1,2, \ldots\right\rangle^{G}$.

ДокАЗАТЕльство. Соотношения группы $G$ обеспечивают очевидное включение

$$
W=\left\langle\left[a, a^{b^{i}}\right], i=1,2, \ldots\right\rangle^{G} \subset \gamma_{\omega} G .
$$

Докажем обратное включение. Для этого достаточно доказать, что

$$
G / W=F_{2} /\left\langle\left[a, a^{b^{i}}\right], i=1,2, \ldots\right\rangle^{F_{2}}
$$

нильпотентно аппроксимируема

По лемме 1 имеем $H=F_{2} /\left\langle\left[a, a^{b^{i}}\right], i=1,2, \ldots\right\rangle^{F_{2}}=F_{2} /\left\langle\left[a, a^{g}\right]\right\rangle^{F_{2}}$. Данная группа $H$ является свободным абелевым расширением:

$$
1 \rightarrow\langle a\rangle^{F_{2}} / \gamma_{2}\langle a\rangle^{F_{2}} \rightarrow F_{2} / \gamma_{2}\langle a\rangle^{F_{2}} \rightarrow F_{2} /\langle a\rangle^{F_{2}}(=\mathbb{Z}) \rightarrow 1
$$

Работа выполнена при поддержке Российского фонда фундаментальных исследований (грант № 02-01-00014).

${ }^{1}$ Мы будем использовать стандартные обозначения $a^{b}=b^{-1} a b,[a, b]=a^{-1} b^{-1} a b$. 
Для свободных абелевых расширений имеется критерий Хартли [3] нильпотентной аппроксимируемости: пусть $F$ - свободная группа ранга $\geqslant 2, N$ - ее нормальная подгруппа, тогда следующие условия эквивалентны:

1) $F / \gamma_{2} N$ нильпотентно аппроксимируема;

2) пусть $\mathfrak{g}$ - аугментационный идеал группового кольца $\mathbb{Z}[F / N]$, тогда $\bigcap_{i=1}^{\infty} \mathfrak{g}^{i}=0$;

3 ) либо $F / N$ аппроксимируется нильпотентными группами без кручения, либо $F / N$ дискриминирует класс нильпотентных групп некоторого конечного примарного $p$-периода.

Так как $F_{2} /\langle a\rangle^{F_{2}}=\mathbb{Z}$ сама является нильпотентной группой без кручения, получаем нильпотентную аппроксимируемость требуемой группы.

Лемма 3. $\gamma_{\omega} G=\gamma_{\omega+1} G$.

ДокАЗАТЕЛЬСтво. Имеем $\left[a, a^{b^{i}}\right]=\left[a,\left[a, a^{b^{i+1}}\right]\right] \in \gamma_{\omega+1} G$. Утверждение следует из леммы 2 и того факта, что элементы трансфинитных нижних централшных рядов нормалшны в группах.

ЗАмечАниЕ. Группа $G / \gamma_{\omega} G$ изоморфна декартову сплетению $\mathbb{Z} w r \mathbb{Z}$, может быть представлена как подгруппа в $G L_{2}(\mathbb{R})$, порожденная $\left(\begin{array}{ll}1 & 1 \\ 0 & 1\end{array}\right),\left(\begin{array}{ll}\xi & 0 \\ 0 & 1\end{array}\right)$ для трансцендентного $\xi$, и не является конечно-представленной по теореме Баумслага [4].

Введем следующие группы:

$$
E_{n}=\left\langle a, b \mid\left[a, a^{b^{i}}\right]=\left[a, a^{a^{b^{i+1}}}\right] ; i \leqslant n\right\rangle,
$$

которые при росте $n$ "приближаются" к рассматриваемой группе $G$. Соотношения $R_{n}$ групп $E_{n}$ задают градуировку соотношений группы $G$, таким образом, $w=1$ в $G$ для некоторого слова $w \in F_{2}$ тогда и только тогда, когда $w=1$ в $E_{l}$ для некоторого $l$.

Лемма 4. В әруппе $E_{n} / \gamma_{2 n+4} E_{n}$ әлемент $\left[a, a^{b}\right]$ нетривиален.

Проблема равенства слов в конечно-порожденных нильпотентных группах алгоритмически разрешима [5], [6]. Алгоритм основан на приведении определяющих соотношений группы и проверяемого слова к некоторому стандартному виду, например, с помощью базиса Холла. Доказательство леммы 4 также сводится к приведению определяющих соотношений групп $E_{n}$ к стандартному виду по модулю $\gamma_{2 n+4} E_{n}$, после которого утверждение становится очевидньм. Лемму 4 , вероятно, также можно доказать, построив вложения групп $E_{n} / \gamma_{2 n+4} E_{n}$ в группы унитреугольных матриц над цельми числами.

Теперь несложно доказать основное утверждение теоремы.

ДокАЗАТЕЛЬСтво. По лемме 3 группа $G$ имеет длину $\omega$, следовательно, для доказательства теоремы достатоно показать, что $\gamma_{\omega} G$ не представима в виде объединения невидимых подгрупп. Предположим противное. Рассмотрим невидимую подгруппу $K$, содержащую элемент $\left[a, a^{b}\right]$. Пусть $K$ нормально порождается элементами $w_{1}, \ldots, w_{s}$ для некоторого $s$. Так как $K$ невидима, то вьполнены следующие включения: $w_{1}, \ldots, w_{s} \in\left\langle\left[w_{1}, a\right],\left[w_{1}, b\right], \ldots,\left[w_{s}, a\right],\left[w_{s}, b\right]\right\rangle^{G}$. Тогда существует $n$ такое, что данные соотношения и включение $\left[a, a^{b}\right] \in\left\langle w_{1}, \ldots, w_{s}\right\rangle$ выполнены и в $E_{n}$, откуда следует, что $\left[a, a^{b}\right] \in \gamma_{\omega} E_{n}$, чего не может быть по лемме 4 .

\section{СПИСОК ЛИТЕРАТУРЫ}

[1] J. Levine // Comment. Math. Helv. 1989. V. 64. № 2. P. 236-255. [2] J. Levine // Israel J. Math. 1991. V. 73. № 1. P. 57-64. [3] B. Hartley // Group Theory. London: Academic Press, 1984. P. 6-120. [4] G. Baumslag// Math. Z. 1961. V. 75. P. 22-28. [5] R. C. Lyndon // Proc. Amer. Math. Soc. 1952. V. 3. P. 579-583. [6] А. И. Мальцев // Матем. сб. 1955. Т. 37 (79). № 3. C. $567-572$.

Математический институт

Принято редколлегией им. В.А. Стеклова РАН 23.09 .2002

E-mail: rmikhailov@mail.ru 